\title{
Cerebral haemodynamics in preterm infants after exposure to dexamethasone
}

\author{
Adelina Pellicer, Francisco Gayá, Tom A Stiris, José Quero, Fernando Cabañas
}

\begin{abstract}
Aim-To determine changes in brain haemodynamics produced by dexamethasone; to evaluate the pathophysiological conditions involved in the effect of dexamethasone.

Methods-A prospective study was made of 12 ventilated preterm infants who received dexamethasone $(0.25 \mathrm{mg} / \mathrm{kg} / 12$ hours) for ongoing chronic lung disease or extubation failure. Cerebral blood flow (CBF), absolute cerebral blood volume (CBV), and cerebral blood volume changes $(\triangle \mathrm{CBV})$ were estimated by near infrared spectroscopy, before and 10, 30, $60,120,180$ and 240 minutes after the first, third, and fifth doses of dexamethasone. All patients were monitored continuously using pulse oximetry, transcutaneous blood gases, and blood pressure.

Results-There were significant short
\end{abstract} term changes in $\triangle \mathrm{CBV}$ on each day of the study; $\triangle \mathrm{CBV}$ increased significantly at $\mathbf{2 4 0}$ minutes compared with values before the first dose, and from 120 minutes onward during the third and fifth doses. However, mean CBV values averaged over 240 minutes after the first, third, and fifth doses did not vary. Mean CBF values averaged over 240 minutes increased progressively up to the fifth dose (significant differences between the first and fifth dose). The short term changes in CBF consisted of a significant increase 60 minutes after dexamethasone administration compared with the before and $\mathbf{1 0}$ minute values in every study. Blood pressure was significantly higher in the third and fifth doses than in the first dose. Blood pressure showed no short term changes. There was no correlation between CBF and blood pressure changes. $\mathrm{TcPCO}_{2}$ (transcutaneous $\mathrm{PCO}_{2}$ ) decreased significantly throughout the study period, with the average mean value in the fifth dose significantly lower than in the first dose. Nevertheless, no short term changes in $\mathrm{TcPCO}_{2}$ were observed.

Conclusions-Postnatal systemic dexamethasone administration produced significant changes in cerebral haemodynamics that seemed to be related to both a direct effect on regional vessel walls and the cumulative effect of dexamethasone.

(Arch Dis Child Fetal Neonatal Ed 1998;79:F123-F128)

Keywords: dexamethasone; cerebral haemodynamics; near infrared spectroscopy
The use of systemic dexamethasone to prevent chronic lung disease in tiny preterm babies is widespread. ${ }^{1-4}$ Increasingly, dexamethasone is used soon after birth $^{56}$ under the assumption that early administration may reduce lung injury at a time when the infant is most exposed to oxygen and barotrauma. ${ }^{6}$ However, we are concerned about the undesirable effects of systemic dexamethasone, particularly on blood pressure, ${ }^{7}$ and the possibility that dexamethasone, as a phospholipase A inhibitor that blocks arachidonic acid metabolism, could have important circulatory effects on different vascular territories. This motivated us to investigate the haemodynamic consequences of systemic dexamethasone on the central nervous system. Initially, we studied the effects of dexamethasone on the brain ${ }^{8}$ and eye ${ }^{9}$ in newborn pigs and found that dexamethasone increased cerebral and retinal blood flow according to radiolabelled microspheres during normoxia. Our next step was to study preterm infants who received dexamethasone to prevent chronic lung disease,${ }^{10}$ in whom we found a significant increase in cerebral and ocular blood flow velocity after they received dexamethasone. This led us to speculate about the potentially harmful effect of dexamethasone on the brain and eye.

In the present study we used the same study protocol as in earlier studies ${ }^{10}$ except that we used near infrared spectroscopy (NIRS). This technique allows several brain haemodynamics that may show parallel changes, depending on pathophysiological conditions, to be studied. This study proposed to clarify the mechanisms by which dexamethasone exerts its effect on brain circulation.

\section{Methods}

The 12 neonates studied had no congenital malformations or sepsis; the indication for dexamethasone was based on abnormal chest radiographs and ventilator weaning failure, as determined by the attending neonatologist. Six intravenous doses of dexamethasone, 0.25 $\mathrm{mg} / \mathrm{kg}$ every 12 hours, were given in the first three days of treatment, after which the dose was tapered off. Written informed parental consent, approved by the Human Studies Committee of the La Paz University Hospital, was obtained for all infants before entry in the study.

Near infrared spectroscopy is based on continuous spectrophotometric measurement of oxygen dependent changes in the absorption properties of the chromophores haemoglobin and cytochrome aa3 in the near infrared range. ${ }^{11}$ 
Assuming a modification in the BeerLambert law, changes in oxyhaemoglobin $\left(\mathrm{O}_{2} \mathrm{Hb}\right)$, deoxyhaemoglobin $(\mathrm{HHb})$, and oxidised cytochrome aa3 (Cytaa3) concentrations (c) $(\mathrm{mM} / \mathrm{l})$ can be obtained from changes in light absorption (A) (optical density):

$\mathrm{A}=$ ecd where $\mathrm{e}$ is the extinction coefficient

of the chromophore $(\mathrm{mM} / \mathrm{cm})$ and $\mathrm{d}$ is the optical path length $(\mathrm{cm})$.

In a medium like the brain, which produces intense scattering, the optical path length is longer than the distance between the points where light enters and leaves the tissue, and $d$ has to be multiplied by a factor (path length factor). The extinction coefficients of $\mathrm{O}_{2} \mathrm{Hb}$ and $\mathrm{HHb}$ are known, ${ }^{12}$ so quantitative changes in the concentration of both chromophores can be calculated from changes observed in light attenuation at several wavelengths. The sum of the changes in $\mathrm{O}_{2} \mathrm{Hb}$ and $\mathrm{HHb}$ show the changes in total haemoglobin concentration $(\Delta \mathrm{THb})$.

The NIRS equipment used was the commercial prototype Critikon Cerebral RedOX Monitor. The sensor is connected to the monitor with an electro-optic cable that contains electrical wires and a flexible fibre optic core consisting of a bundle of individual glass fibres. Light pulses from a laser in the monitor are transmitted through the fibre bundle to the sensor head (emission window). Light pulses emerging from the tissue are detected by a single photodiode housed in the sensor head. The signals from the sensor head are carried to the monitor by a shielded pair of electrical cores within the electro-optic cable. The laser diodes produce sequential pulses of monochromatic light, maintaining a precise spectral performance at factory calibrated wavelengths of $776.5,819.0,871.4$ and $908.7 \mathrm{~nm}$. Absorption of these four wavelengths is continuously sampled at a rate of $2 \mathrm{~Hz}$. The haemoglobin absorption coefficients $\left(\mathrm{O}_{2} \mathrm{Hb}\right.$ and $\left.\mathrm{HHb}\right)$ used were derived directly from absorption spectra data published by Wray et al. ${ }^{12}$ Wavelength dependent scattering factors are incorporated into the inverse matrix. The values of the wavelength dependent scattering factors were calculated by fitting a polynomial curve to the data of Essenpries et $a l^{13}$ and Thorniley et al. ${ }^{14}$ In the present study a fixed path length factor of $4.4^{15}$ was used. Quantified chromophore concentration data are presented in micromolar units. The sensor was placed frontally in all patients.

CALCULATION OF CHANGES IN $\triangle \mathrm{CBV}$

This parameter may be continuously estimated by NIRS, because $\Delta \mathrm{THb}(\mu \mathrm{mol} / \mathrm{l})$ is proportional to $\triangle \mathrm{CBV}(\mathrm{ml} / 100 \mathrm{~g})$, using the equation $^{16}$ :

\section{$\Delta \mathrm{CBV}=\mathrm{K} \cdot \Delta \mathrm{THb} / \mathrm{H}$}

where the value of the constant $\mathrm{K}, 0.89$, is obtained from the molecular weight of haemoglobin (64500), brain tissue density $(1.05 \mathrm{~g} / \mathrm{m} 1)$, the large vessel:tissue ratio, which is assumed to be equal to $0.69,{ }^{17}$ and decimal conversions. $\mathrm{H}$ is the haemoglobin concentration in a large vessel blood sample. In the present study $\Delta \mathrm{THb}$ was calculated as the difference between the baseline value (related to an arbitrary zero) and the mean value of all samples obtained in the 20 second period before each measurement defined in the study protocol.

MEASUREMENT OF CBV

To quantify CBV in absolute terms we used the method of Wyatt et al, ${ }^{18}$ which is based on the indicator-dilution principle. When the infant is stable and $\mathrm{CBF}$ and the cerebral metabolic oxygen rate are constant, $\mathrm{O}_{2} \mathrm{Hb}$ is used as a tracer by increasing the oxygen content in inspired air. The small increase in saturation that results and the parallel decrease in $\mathrm{HHb}$ are used to measure total cerebral haemoglobin, which is proportional to CBV.

To quantify CBV we changed the inspired oxygen fraction by $10-20 \%$ for $2-5$ minutes so that the tracer was at a steady state and balanced throughout the brain:

$\mathrm{CBV}=\mathrm{K} \cdot \Delta\left(\mathrm{O}_{2} \mathrm{Hb}-\mathrm{HHb}\right) / 2 \cdot \Delta \mathrm{SaO}_{2} \cdot \mathrm{H}$ where $\mathrm{K}$ and $\mathrm{H}$ are as defined above, and $\Delta \mathrm{SaO}_{2}$ is the change in haemoglobin saturation.

MEASUREMENT OF CBF

Using $\mathrm{O}_{2} \mathrm{Hb}$ as an intravascular tracer, $\mathrm{CBF}$ was calculated, based on the Fick principle. ${ }^{19-21}$ A sudden small change in $\mathrm{O}_{2} \mathrm{Hb}$ was induced by giving the patient a higher inspired fraction of oxygen for several breaths, which caused $\mathrm{SaO}_{2}$ to rise rapidly by about $5 \%$. CBF was calculated from the changes in both parameters recorded over a $6-8$ second period: $\mathrm{CBF}=\mathrm{K} \cdot \Delta\left(\mathrm{O}_{2} \mathrm{Hb}-\mathrm{HHb}\right)$

$2 \cdot \mathrm{H} \cdot$ integral $[0 \rightarrow \mathrm{t}]$ of $\Delta \mathrm{SaO}_{2}$ where $\mathrm{K}$ and $\mathrm{H}$ have been defined.

Before measuring cerebral blood flow $\left(\mathrm{O}_{2} \mathrm{Hb}-\mathrm{HHb}\right)$ and $\mathrm{SaO}_{2}$ should be stable for a period longer than the maximal cerebral transit time. The analysis of the NIRS curves, including quality check of the raw data and rejection of the measurement or calculation of $\mathrm{CBF}$, were made off-line using a custom made computer program and an algorithm that has been published before. ${ }^{22}$

STUDY PROTOCOL

After a period of stabilisation, the first NIRS measurements were used as baseline values (DEX-0). Dexamethasone was administered as an intravenous bolus and NIRS measurements were repeated at 10 (DEX-10), 30 (DEX-30), 60 (DEX-60), 120 (DEX-120), 180 (DEX180) and 240 (DEX-240) minutes. Because of limitations in the procedure, specifically the steady state recovery time of at least 5 minutes between measurements, CBF was measured one to three (median one) times 15-30 minutes before drug administration, once for DEX-10, and one to three times (median one) for every other period. Absolute CBV was

Table 1 Characteristics and clinical data of infants studied (median and 25th-75th percentile)

Birthweight (g)

$920(721-1117)$

Gestational age (weeks)

Male:female

Age at dose 1 dexamethasone (days)

Weight at dose 1 dexamethasone $(\mathrm{g})$ $28(26-28)$

$10(9-21)$ Mortality and age (days)

$1 / 12$ (48) 

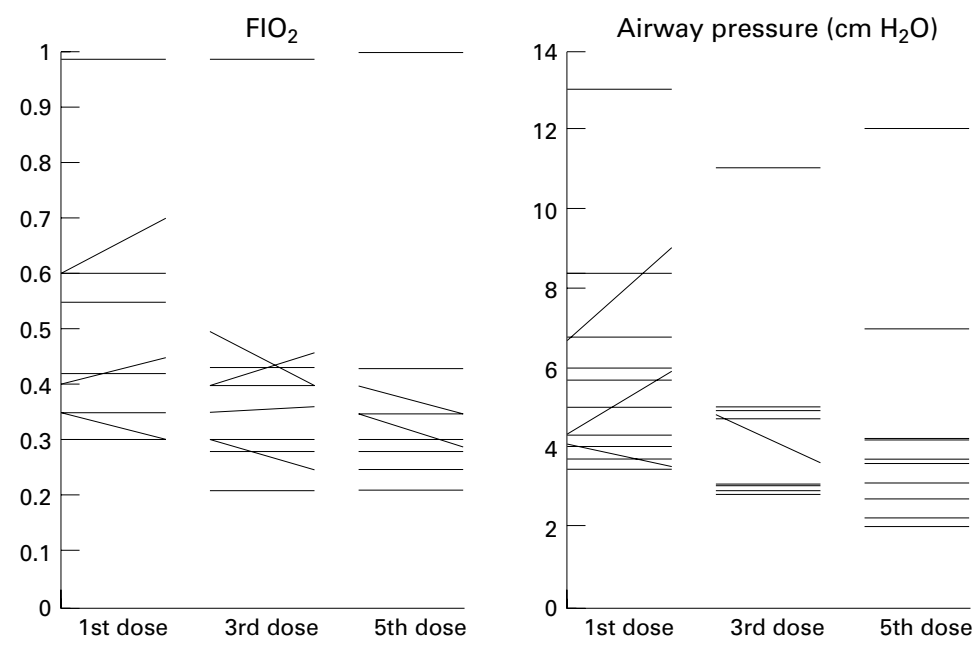

Figure 1 Inspired oxygen fraction and mean airway pressure of the patients at the beginning (DEX-0) and at the end (DEX-240) of the study during the first, third, and fifth dose of dexamethasone.

quantified once 5 minutes before dexamethasone administration, and once at each measurement period. Every day of the study (during the first, third, and fifth dose) $\triangle \mathrm{CBV}$ was estimated continuously in relation to baseline value (DEX-0), an arbitrary zero.

All NIRS studies were performed by the same investigator (AP). No patient had received medication likely to affect cerebral circulation. Every measurement was made with the patient in a quiet state and under stable oxygenation, ventilation, and haemodynamic conditions. For the more than 4 hours that each study period lasted, patients remained undisturbed, in the same position, and did not receive medication. Tracheal aspiration was carried out 1 hour before dexamethasone and when the NIRS study concluded 4 hours later.

Before beginning treatment with dexamethasone and after every NIRS study, a complete cerebral ultrasound scan was performed to detect changes in parenchymal echogenicity or periventricular-intraventricular haemorrhage. All cerebral ultrasound studies were performed by the same investigator (FC).

Patients were monitored continuously for heart rate, breathing rate, transcutaneous $\mathrm{PCO}_{2}$ and $\mathrm{PO}_{2}$. Arterial oxygen saturation was measured on every pulse beat by pulse oximetry (Fas Trac, Critikon). Blood pressure was measured by oscillometry (Dynamap, Critikon inc; Tampa, FL, USA) at each measurement period defined above. Total haemoglobin con-

Table 2 Mean (SEM) values averaged over 240 min for the first, third, and fifth doses of dexamethasone. In parenthesis: mean baseline values before dexamethasone

\begin{tabular}{llllll}
\hline & $\begin{array}{l}\text { Before dexamethasone } \\
\text { (baseline) }\end{array}$ & First dose & Third dose & Fifth dose & p Value $^{a}$ \\
\hline $\mathrm{CBF}$ & $(22.9(2.0))$ & $31.1(2.2)$ & $33.7(1.9)$ & $36.9(1.6)^{\mathrm{b}}$ & 0.0024 \\
$\mathrm{CBV}$ & $(2.3(0.2))$ & $3.2(0.2)$ & $3.3(0.2)$ & $3.2(0.2)$ & NS \\
$\mathrm{MAP}$ & $(47(3))$ & $50(1)$ & $56(1)^{\mathrm{c}}$ & $56(1)^{\mathrm{c}}$ & 0.001 \\
$\mathrm{HR}$ & $(155(3))$ & $154(1.5)$ & $140(2)^{\mathrm{c}}$ & $144(2.2)^{\mathrm{c}}$ & 0.001 \\
$\mathrm{TcPCO}_{2}$ & $(67.6(10.6))$ & $56.9(2.3)$ & $52.2(1)$ & $47.6(0.8)^{\mathrm{b}}$ & 0.001 \\
\hline
\end{tabular}

CBF: cerebral blood flow, CBV: cerebral blood volume, MAP: mean arterial blood pressure, HR: heart rate, $\mathrm{TcPCO}_{2}$ : transcutaneous $\mathrm{PCO}_{2}$. The values for each variable were calculated by averaging the values measured at $0,10,30,60,120,180$ and $240 \mathrm{~min}$ for the first, third, and fifth doses of dexamethasone administered.

Analysis of variance (ANOVA) of two factors for repeated measurments.

${ }^{b} \alpha=0.05$ versus first dose (Bonferroni test).

$\alpha=0.01$ versus first dose (Bonferroni test). centration was measured every day of the study using a Coulter counter.

NIRS and other data were stored on magnetic disc for later analysis.

STATISTICAL ANALYSIS

Data were analysed using the BMDP-5V module of the BMDP Classic Release 7.0 program (Statistical Software). ${ }^{23}{ }^{24}$ Quantitative data are given as mean (SEM).

To study the evolution of the variables throughout the study, two sources of variation (factors) were assumed: (a) the effect of the cumulative dose (dose factor) by days of treatment (changes between doses); and (b) variation over the 240 minute period (time factor) after administering each dose (intradose or short term changes). We analysed the effect of each factor independently and determined if there was interaction or synergy between them. Two way analysis of variance for repeated measures, factoring for time and dose, was used to study their effect on the following parameters: CBF, CBV, blood pressure, heart rate, and $\mathrm{PCO}_{2}$. Three doses (first, third, and fifth doses) and seven times (DEX-0, DEX-10, DEX-30, DEX-60, DEX-120, DEX-180, DEX-240) were considered. Firstly, we studied the main effects of each factor-that is, the behaviour of the average values obtained for each dose, or the average value of the three doses for each time. In the case of interaction between the two factors, the individual effects of time within each dose were also analysed. In this study interaction was interpreted as the difference between the profiles of the times within each dose. We used logarithmic transformation to study the significance of changes in $\mathrm{CBF}$.

For $\triangle \mathrm{CBV}$, we analysed only the effect of time for each dose because the cumulative effect of dose could not be evaluated as it is a relative value that cannot be compared between doses. A post hoc test for pairwise comparisons was carried out using the Bonferroni procedure. ${ }^{25} 26$ A correlation analysis was developed to examine the relation between blood pressure and $\mathrm{CBF}$ measurements. $\mathrm{p}$ values $<0.05$ were considered significant.

\section{Results}

The patients' characteristics and clinical data are shown in table 1 . The evolution of the respiratory profile, as expressed by the intradose and interdose supplementary oxygen requirements and mean airway pressure, is shown in fig 1 . The mean values averaged over $240 \mathrm{~min}-$ utes for CBV, CBF, blood pressure, heart rate, and $\mathrm{TcPCO}_{2}$ after the first, third, and fifth doses of dexamethasone and mean baseline values (before dexamethasone) are shown in table 2 .

There was a significant increase in blood volume after dexamethasone administration. The short term changes in $\triangle \mathrm{CBV}$ for each day of study are shown in fig 2 . Post hoc analysis disclosed differences, as a function of time for each dose with respect to DEX-0 values, 240 minutes after dexamethasone for the first dose, and from 120 minutes onwards during the 
third and fifth doses (fig 2). However, mean CBV values averaged for 240 minutes after the first, third, and fifth doses did not vary significantly (table 2).

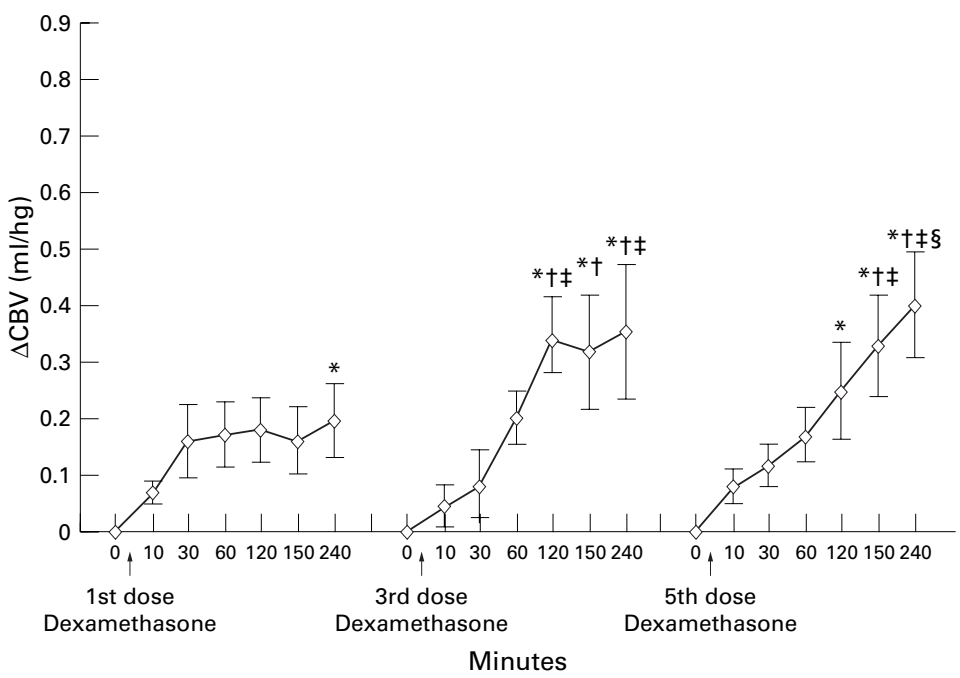

Figure 2 Mean values ( $\mathrm{ml} / \mathrm{hg}$ ) of $\triangle \mathrm{CBV}$ as a function of time during the first, third, and fifth doses of dexamethasone. * vs DEX-0; +vs DEX-10; vs DEX-30; Jvs DEX-60 (a $=0.05$, Bonferroni test .
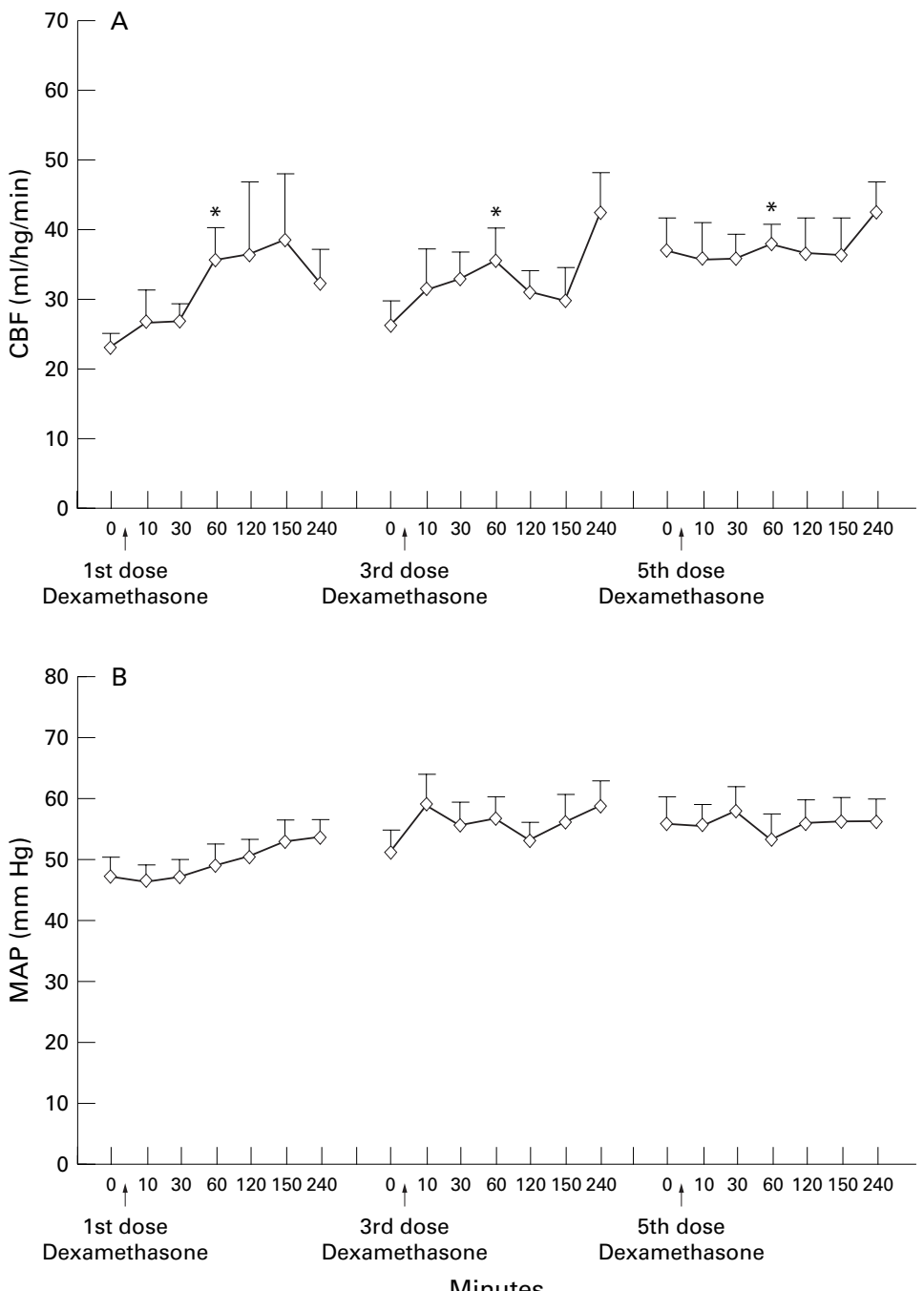

Figure 3 Mean values of $C B F(A)$ and blood pressure $(M A P)$ (B) as a function of time during the first, third, and fifth doses of dexamethasone ${ }^{\star}$ vs DEX-0;+vs DEX-10.
Mean CBF values averaged for 240 minutes increased progressively up to the fifth dose (table 2). Post hoc pairwise comparisons showed significant changes between the first and fifth doses. In every study the short term changes in $\mathrm{CBF}$ were similar, with a significant increase 60 minutes after dexamethasone in relation to DEX-0 and DEX-10 values (fig 3).

The physiological variables blood pressure, heart rate, and $\mathrm{PCO}_{2}$ also varied across the entire study period (table 2). Blood pressure values during the third and fifth doses were significantly higher than in the first dose, whereas heart rate was significantly lower in the third and fifth doses than in the first dose. The short term changes in blood pressure showed no significant differences on any study day (fig 3). There was no correlation between $\mathrm{CBF}$ and blood pressure changes.

$\mathrm{TcPCO}_{2}$ decreased significantly throughout the study period; the averaged mean value during the fifth dose was significantly lower than during the first dose. Although there were also short term changes in $\mathrm{TcPCO}_{2}$, which was lower at DEX-240 than at DEX-0, the differences were not significant.

We found no interaction between the effect of dose and time in our analysis of the behaviour of these variables.

Cerebral ultrasound findings before and during dexamethasone treatment, and 40 weeks after conception were: periventricularintraventricular haemorrhage grades I-III in eight patients; abnormally increased periventricular echogenicity in six patients, four of whom also had periventricular-intraventricular haemorrhage; and two patients with normal cerebral ultrasound scans. No patient had clinically significant sonographic changes during dexamethasone treatment. At 40 weeks after conception, seven patients had normal sonograms; three had persistent periventricular echogenicity, one with mild ventricular dilation; one with posthaemorrhagic hydrocephalus had a ventriculoperitoneal shunt; and the remaining patient, who died at 48 days, showed a persistent increase in periventricular echogenicity at 30 days of life.

Complications that could be related to steroid use in our patients were hypertension (three cases) and necrotising enterocolitis (one case). Three patients required laser treatment for severe retinopathy of prematurity.

\section{Discussion}

As in our earlier study, ${ }^{10}$ we found significant changes in several haemodynamic parameters. The behaviour of CBV was analysed from two vantage points: intradose effects by continuous monitoring of changes in $\mathrm{THb}$ after dexamethasone administration $(\triangle \mathrm{CBV})$, and the cumulative effect of dexamethasone by comparing CBV changes throughout the study period. Changes in $\mathrm{CBF}$ were studied within each dose and in response to repeated doses of dexamethasone.

Although this and our earlier study ${ }^{10}$ had the same design, the two study populations were not comparable because of limitations in the techniques. In our experience the Doppler 
probe applied over the anterior fontanelle modifies NIRS traces, causing artefacts to be recorded, which makes results unreliable. This effect probably has to do with interruption of the venous drainage in the sagittal sinus. To avoid this undesirable effect of the method, we evaluated the second population only by NIRS to verify if the changes in cerebral blood flow velocity and resistance index observed in our first study corresponded with changes in CBF, as determined by NIRS, in the present study.

In this study dexamethasone had substantial effects on cerebral haemodynamics. The short term changes in $\triangle \mathrm{CBV}$ suggest drug induced cerebral vasodilation, which increased vascular volume and lowered cerebrovascular resistance. This observation was coupled with a significant increase in CBF 60 minutes after dexamethasone administration.

An analysis of the behaviour of such haemodynamic parameters throughout the study showed that CBV remained within a similar range, but that $\mathrm{CBF}$ values increased progressively up to the fifth dose.

These NIRS findings concur with the Doppler changes found in our previous study, ${ }^{10}$ indicating that dexamethasone seems to have a direct effect on the vessel wall. In our Doppler study the short term changes in cerebral blood flow velocity were similar to the $\mathrm{CBF}$ changes seen in the NIRS study. Moreover, the cerebral blood flow velocity changes throughout the Doppler study period concurred with the CBF changes observed in the NIRS study. An inverse variation in the resistance index was also observed, indicating possible vasodilation of the vessels investigated. On the other hand, the short term changes in $\triangle \mathrm{CBV}$ increased progressively after each dexamethasone dose and reached their highest values later than $\mathrm{CBF}$, which could be attributed to a more prolonged and/or potent vasodilator effect on the venous compartment than on the arteriolar-capillary territory. The transmission of positive pressures to intrathoracic structures may have important effects on $\triangle \mathrm{CBV}$. Leahy et $a l^{7}$ showed that the increase in CBV with intermittent positive pressure ventilation was proportional to lung compliance. In our patients fractional inspired oxygen concentration and mean airway pressure requirements remained unchanged for most of the patients on every day of the study (fig 1). At the time, intradose $\mathrm{PCO}_{2}$ changes were not significant. These two facts precluded a significant improvement in lung mechanics, and an increase in transpulmonary pressures impeded venous drainage, contributing to the increase in $\triangle \mathrm{CBV}$ observed in the 4 hours after every dexamethasone dose. Nevertheless, a positive effect on lung compliance was described when respiratory performance beyond the first day of dexamethasone treatment was compared with pre-dexamethasone values. ${ }^{28}$

Another important aspect to consider is that NIRS serves to analyse haemodynamic changes that correspond to a representative portion of brain, but regional variations cannot be excluded. In fact, in our previous report using colour Doppler flow imaging, ${ }^{10}$ we found a similar trend in the behaviour of cerebral blood flow velocity, ocular blood flow velocity, and the resistance index, but the changes were less dramatic in the ophthalmic artery than in the anterior cerebral artery or carotid artery.

In both the NIRS and Doppler studies we found a significant increase in blood pressure. However, neither of the studies showed any association between changes in arterial pressure and changes in cerebral haemodynamic parameters. Moreover, in this study short term changes in $\mathrm{CBF}$ did not correlate with blood pressure changes. We feel that this finding reinforces the idea of a direct vascular effect which modifies brain circulation and seems to be independent of the changes exerted by dexamethasone on blood pressure.

The changes in cerebral haemodynamic parameters observed in the present study cannot be attributed to blood gas variations (table 2) because $\mathrm{TcPCO}_{2}$ tended to decrease throughout the study period; such a decrease would have had an opposite effect on the haemodynamic variables evaluated. ${ }^{16}{ }^{29} 30$

In an experimental study in piglets ${ }^{9}$ we found a significant increase in retinal blood flow during normoxia after dexamethasone administration, compared with a control group. Vitreous prostanoids and leucotrienes were significantly lower after dexamethasone infusion. The changes observed in cerebral circulatory parameters after dexamethasone could be related to the effect of the drug on arachidonic acid metabolism because dexamethasone is an inhibitor of phospholipase A and therefore reduces prostanoids, leucotrienes, and free radical generation, which regulate vasomotor tone physiologically. However, other pharmacological effects of dexamethasone may be involved, resulting in a predominant vasodilator effect. ${ }^{31-34}$ Thus dexamethasone increases the release of natriuretic peptides, ${ }^{31}{ }^{32}$ stabilises cell membranes, and inhibits free oxygen radical reactions, ${ }^{33}$ all properties that may promote vasodilation.

The changes observed in brain haemodynamics after systemic administration of steroids are of concern, particularly when these drugs are given soon after birth, because the risk of developing intracranial haemorrhage in an immature brain is theoretically greater. In our opinion, these findings do not contradict earlier reports of the beneficial effects of reducing periventricular haemorrhage by prenatal steroid administration. ${ }^{35}{ }^{36}$ Prenatal steroids improve cardiovascular function in preterm births, ${ }^{37}{ }^{38}$ thus protecting the brain against the undesirable effects of haemodynamic instability. ${ }^{39}$

In summary, our study showed substantial changes in brain circulation after systemic dexamethasone, which concurs with our findings in studies in neonatal animal models ${ }^{89}$ and in human preterm infants..$^{10}$ These changes indicate that dexamethasone has a direct vasodilator effect. Prospective studies should be conducted to test the impact of dexamethasone using different protocols. Likewise, given the vulnerability of the immature brain to ischaemic and haemorrhagic lesions in the first days 
of life, early dexamethasone administration merits particular attention.

This study was supported by grant from the Fondo de Investiación Sanitaria (FIS 94/0198).

We thank Rosario Madero, from the Research Unit of La Paz University Hospital, for her valuable cooperation in statistics.

Thiversity Hospital, for her valuable cooperation in statistics.
This study was presented, in part, at the European Society for

Pediatric Research, Annual Meeting, Alicante, Spain, September, 1995.

1 Avery GB, Fletcher AB, Kaplan M, Brudno DS . Controlled trial of dexamethasone in respiretor-dependent infants with bronchopulmonary dysplasia. Pediatrics 1985;75:10611 .

2 Collaborative Dexamethasone Trial Group . Dexamethasone therapy in neonatal crhonic lung disease: an international placebo-controlled trial. Pediatrics 1991;88:421-7.

3 Cummings JJ, D'Eugenio DB, Gross SJ. A controlled trial of dexamethasone in preterm infants at high risk for bronchopulmonary dysplasia. N Engl F Med 1989;320:1505-10

4 Mammel MC, Green TP, Johnson DE, Thompson TR. Controlled trial of dexamethasone therapy in infants with bronchopulmonary dysplasia. Lancet $1983 ; \mathbf{i}: 1356-8$.

5 Rastogi A, Morales P, Akintori S, Bez M, David R, Pildes RS. Randomized double blind trial of dexamethasone (D) plus surfactant (S) for prevention of BPD. Pediatr Res plus surfactan

6 Yeh TF, Torre JA, Rastogi A, Anyebuno MA, Pildes RS Early postnatal dexamethasone therapy in premature
infants with severe respiratory distress syndrome: a infants with severe respiratory distress syndrome: a

$7 \mathrm{Ng}$ PC. The effectiveness and side effects of dexamethasone in preterm infants with bronchopulmonary dysplasia. Arch Dis Child 1993;68:330-6.

8 Stiris TA, García-Alix A, Blanco D, Cabañas F, Pellicer A Quero J. The response of total and regional blood flow to dexamethasone (DEX) infusion in newborn piglets during normoxia. Pediatr Res 1994;35:269A

9 Stiris TA, Blanco D, Codoceo R, et al. Effects of dexamethasone on retinal and choroidal blood flow during normoxia and hyperoxia in newborn piglets. Pediatr Res 1996;40:592-6.

10 Cabañas F, Pellicer A, García-Alix A, Quero J, Stiris TA. Effect of dexamethasone therapy on cerebral and ocular blood flow velocity in premature infants studied by colour blood flow velocity in premature infants studied by

11 Jobsis FF. Noninvasive infrared monitoring of cerebral and myocardial oxygen sufficiency and circulatory parameters. myocardial oxygen suffici

12 Wray S, Cope M, Delpt DT, Wyatt JS, Reynolds EOR. Characterisation of the near infrered absorption spectra of cytochrome aa 3 and haemoglobine for non-invasive monitoring of cerebral oxygenation. Biochim Byiophys Acta 1988;933:184-92.

13 Essenpries M, Cope M, Elwell CE, Arridge SR, van de Zee P, Delpy DT. Wavelength dependence of the differentia pathlength factor and the long slope in the time resolved tissue spectroscopy. In: Dirnagl U, ed. Optical Imaging of Brain Function and Metabolism. New York:Plenum Press, 1993: 9-20.

14 Thorniley MS, Lane NJ, Manek S, Green CJ . Noninvasive measurement of respiratory chain dysfunction following hypothermic renal storage and transplantation. Kidney Int 1994; 45:1489-96.

15 Wyatt JS, Cope M, Delpy DT. Measurement of optical pathlength for cerebral near-infrared spectroscopy in newpathlength for cerebral near-infrared spectros
born infants. Dev Neurosci 1990;12:140-4.

16 Wyatt JS, Edwards AD, Cope M, et al. Response of cerebral blood volume to changes in arterial carbon dioxide tension in preterm and term infants. Pediatr Res 1991;29:553-7.

17 Lammertsma AA, Brooks DJ, Bearney RP. In vivo measurement of regional cerebral hemotocrit using possitron emission tomography. $\mathcal{F}$ Cereb Blood Flow Metab 1984;4:317-22

18 Wyatt JS, Cope M, Delpy DT, et al. Quantitation of cerebral blood volume in human infants by near-infrared spectroscopy. F Apply Physiol 1990;68:1086-91
19 Edwards AD, Wyatt JS, Richardson D, Delpy DT, Cope M, Reynolds EOR. Cotside measurement of cerebral blood flow in ill preterm infants by near-infrared spectroscopy. Lancet 1988; ii:770-1.

20 Edwards AD, Wyatt JS, Richardson CE, Delpy DT, Cope $\mathrm{M}$, Reynolds EOR. Precision of cerebral blood flow (CBF) measurements by near-infrared spectroscopy (NIRS). Pediatr Res 1989;26:520A.

21 Edwards AD, Wyatt JS, Richardson C, et al. Effects of indometacin on cerebral haemodynamics in very preterm infants. Lancet 1990;335:1491-5.

22 Skov L, Pryds O, Greisen G. Estimating cerebral blood flow in newborn infants: Comparison of near infrared spectros copy and ${ }^{133}$ Xe Clearance. Pediatr Res 1991;30:570-3

23 Dixon WJ, Brown MB, Engelman L, Hill MA, Jennich RI. In: Dixon WJ, ed. BMDP Statistical Software Man

24 Schluchter MD Analysis of incomplete multivariate data using linear models with structured covariance matrices. using linear models with structured

25 Draper N, Smith H. Applied regresion analysis. 2nd Edn. New York: John Wiley, 1981

26 Cochran WG, Cox GM. Experimental designs. 2nd Edn. New York: John Willey, 1990

27 Leahy FAN, Durand M, Cates D, Chernick V. Cranial blood volume changes during mechanical ventilation and spontaneous breathing in newborn infants. $f$ Pediatr 1982;101:984-7.

28 Durand M, Sardesai S, McEvoy C. Effects of early dexamethasone therapy on pulmonary mechanics and chronic lung disease in very low birth weight infants: A randomized, controlled trial. Pediatrics 1995;95:584-90

29 Pryds O, Greisen G, Skov LL, Friis-Hansen B. Carbon dioxide-related changes in cerebral blood volume and cerebral blood flow in mechanically ventilated preterm neonates: comparison of near-infrared spectrophotometry and ${ }^{133}$ xenon clearance. Pediatr Res 1990;27:445-9.

30 Brun NC, Greisen G. Cerebrovascular responses to carbon dioxide as detected by near-infrared spectrophotometry: Comparison of three different measures. Pediatr Res 1994;36:20-4.

31 Gardner DG, Have S, Trachevsky D, Schonk D, Baxter JD. Arterial natriuretic peptide mRNA is regulated by glucocorticoids in vivo. Biochem Biophys Res Commun 1986;139:1047-54

32 Danaberg J, Gekiw RJ.Corticoid regulation of atrial natriuretic factor secretion and gene expression. Am $\mathcal{F}$ Physiol 1992;263:H1377-H181.

33 Demopoulos HB, Flamm ES, Pietronigro DD.The free radical pathology and microcirculation in the major central nervous system disorders. Acta Physiol Scand Suppl 1980;492:91-119.

34 Banglam AD, Standish MM, Weissmann G.The action of steroids and streptolysin $\mathrm{S}$ on the permeability of phospholipid structures to cations. $f$ Mol Biol 1965;13:253-9.

35 Leviton A, Kuban KC, Pagano M, Allred EN, Van Marter L. Antenatal corticosteroids appear to reduce the risk of postnatal germinal matrix hemorrhage in intubated low birth weight babies. Pediatrics 1993;91:1083-8.

36 O'Shea M, Savitz DA, Hage ML, Feinstein KA. Prenatal events and the risk of subependymal/intraventricular hemorrhage in very low birth weight neonates. Paediatr Perinat Epidemiol 1992;6:352-62.

37 Garland JS, Buck R, Leviton A. Effect of maternal glucocorticoid exposure on risk of severe intraventricular hemorrhage in surfactant-treated preterm infants. $\mathcal{F}$ Pediatr 1995;126:272-9.

38 Padbury JF, Polk DH, Hervin MG, Berri LM, Ikegami M, Jobe AH. Postnatal cardiovascular and metabolic rein fetal sheep born prematurely by cesarean section. Pediatr Res 1995;38:709-15.

39 Volpe JJ. Intracranial hemorrhage: Germinal matrixintraventricular hemorrhage of the premature infant. In: Volpe JJ, ed. Neurology of the newborn. 3rd Edn. Philadelphia: W B Saunders, 1995:403-63. 